\title{
Quantum Spins and Quantum Links: The D-Theory Approach to Field Theory
}

\author{
U.-J. Wiese ${ }^{a}$ \\ ${ }^{a}$ Center for Theoretical Physics, Laboratory for Nuclear Science and Department of Physics \\ Massachusetts Institute of Technology (MIT), Cambridge, MA 02139
}

\begin{abstract}
A new non-perturbative approach to quantum field theory is proposed. Instead of performing a path integral over configurations of classical fields, D-theory works with discrete quantized variables. Classical spin fields are replaced by quantum spins, and classical gauge fields are replaced by quantum links. The classical fields of a $d$-dimensional quantum field theory reappear as low-energy effective degrees of freedom of the discrete variables, provided the $(d+1)$-dimensional D-theory is massless. When the extent of the extra Euclidean dimension becomes small in units of the correlation length, an ordinary $d$-dimensional quantum field theory emerges by dimensional reduction. The D-theory formulation of some spin models and gauge theories is constructed explicitly. In particular, QCD emerges as a quantum link model.
\end{abstract}

\section{Introduction}

Field theories are usually quantized by performing a path integral over configurations of classical fields. This is the case both in perturbation theory and in Wilson's non-perturbative lattice formulation of quantum field theory. However, there is another form of quantization, which is well-known from quantum mechanics: a classical angular momentum vector can be replaced by a vector of Pauli matrices. The resulting quantum spin is described by discrete variables $\pm 1 / 2$, while the original classical angular momentum vector represents a continuous degree of freedom. Here we propose to apply this kind of quantization to field theory.

Of course, it is far from obvious that such a quantization procedure is equivalent to the usual one. For example, a single spin $1 / 2$ has the same symmetry properties as a classical angular momentum vector, but it operates in a finite Hilbert space. How can the full Hilbert space of a quantum field theory be recovered when the classical fields are replaced by analogs of quantum spins? Indeed, as we will see, this requires a specific dynamics, which, however, is generic in a wide variety of cases. This includes spin models and gauge theories, and, in particular, QCD. In these cases, a collective excitation of a large number of dis- crete variables acts as a classical field, in the same way as many spins $1 / 2$ can act as a classical angular momentum vector. To collect a large number of discrete variables, it turns out to be necessary to formulate the theory with an additional Euclidean dimension. When the $(d+1)$-dimensional theory is massless, the classical fields emerge as low-energy excitations of the discrete variables. When the extent of the extra dimension becomes small in units of the correlation length, the desired $d$-dimensional quantum field theory emerges via dimensional reduction. Dimensional reduction of discrete variables is a generic phenomenon that occurs in a variety of models, thus leading to a new non-perturbative formulation of quantum field theory, which we call $D$-theory.

The chiral symmetries of fermions have already motivated extensions to five dimensions. In order to solve the lattice fermion doubling problem, Kaplan has proposed to view our 4-d spacetime as a domain wall in five dimensions. A 5-d fermion, which is always vector-like, then develops a zero mode bound to the domain wall, which appears as a chiral fermion from a 4-d point of view. For QCD, Shamir has simplified Kaplan's ideas by working with fixed boundary conditions for the fermions in a 5-d slab. In D-theory, the extra dimension appears for reasons related to the 
bosonic degrees of freedom. Still, as we will see, the bosonic D-theory construction fits very naturally with Shamir's variant of Kaplan's fermion proposal.

D-theory has several interesting features that go beyond Wilson's non-perturbative lattice formulation of quantum field theory. For example, due to the use of discrete variables, the theory can be completely fermionized. All bosonic fields can be written as pairs of fermionic constituents, which we call rishons. The two indices of a bosonic matrix field - for example, the two color indices of a gluon field matrix - can be separated because they are carried by two different rishons. This leads to new ways to attack the large $N$ limit of QCD and other interesting field theories. D-theory is attractive also from a computational point of view. Discrete variables are particularly well suited for numerical simulations using very powerful cluster algorithms. In fact, the first cluster algorithm for a model with a continuous gauge group has been constructed for a $U(1)$ quantum link model, and it is plausible that this construction can be extended to non-Abelian gauge theories.

$S U(2)$ and $U(1)$ quantum link models were written down by Horn as early as 1981 [1], and they were rediscovered and discussed in more detail by Orland and Rohrlich in 1990 [2]. Recently, they have been rediscovered again, and it has been realized how they are related to ordinary gauge theories via dimensional reduction [3]. Quantum link QCD was constructed in [theory was discussed in [5]. A detailed analysis of the $U(1)$ quantum link model is the subject of three contributions to these proceedings [6].

This talk is organized as follows. In section 2, D-theory is explained in the context of the $O(3)$ model. Section 3 contains the D-theory representation of complex vector and matrix fields. This leads to the explicit construction of some models in section 4. The dimensional reduction of quantum links models to ordinary gauge theories is discussed in section 5 , and the inclusion of fermions is described in section 6. Finally, section 7 contains the conclusions.

\section{The $O(3)$ Model from D-Theory}

Let us illustrate the basic ideas behind Dtheory in the simplest example - the 2-d $O(3)$ model, which we view as a Euclidean field theory in $1+1$ dimensions. Like QCD, this model is asymptotically free, and has a non-perturbatively generated mass-gap. In Wilson's formulation of lattice field theory the model is formulated in terms of classical 3-component unit vectors $\vec{s}_{x}$ located on the sites $x$ of a quadratic lattice. The Euclidean action of the model is given by

$S[\vec{s}]=-\sum_{x, \mu} \vec{s}_{x} \cdot \vec{s}_{x+\hat{\mu}}$,

where $\hat{\mu}$ represents the unit vector in the $\mu$ direction. The theory is quantized by considering the classical partition function

$Z=\int \mathcal{D} \vec{s} \exp \left(-\frac{1}{g} S[\vec{s}]\right)$,

which represents a path integral over all classical spin field configurations $[\vec{s}]$. Here $g$ is the coupling constant. Due to asymptotic freedom, the continuum limit of the lattice model corresponds to $g \rightarrow 0$. In this limit the correlation length $\xi \propto \exp (2 \pi / g)$ diverges exponentially. The strength of the exponential increase is given by the 1-loop $\beta$-function coefficient $2 \pi$ of the 2 -d $O(3)$ model.

In contrast to the standard procedure, in Dtheory one does not quantize by integrating over the classical field configurations $[\vec{s}]$. Instead, each classical vector $\vec{s}_{x}$ is replaced by a quantum spin operator $\vec{S}_{x}$ (a Pauli matrix for spin $1 / 2$ ) with the usual commutation relations

$\left[S_{x}^{i}, S_{y}^{j}\right]=i \delta_{x y} \epsilon_{i j k} S_{x}^{k}$.

The classical action of the 2-d $O(3)$ model is replaced by the Hamilton operator

$H=J \sum_{x, \mu} \vec{S}_{x} \cdot \vec{S}_{x+\hat{\mu}}$,

thus, leaving us with a quantum Heisenberg model. Here we restrict ourselves to antiferromagnets, i.e., to $J>0$. Ferromagnets have a conserved order parameter, and therefore require a special treatment. Like the classical action $S[\vec{s}]$, 
the Hamilton operator $H$ is invariant under global $S O(3)$ transformations. In quantum mechanics this follows from $[H, \vec{S}]=0$, where

$$
\vec{S}=\sum_{x} \vec{S}_{x}
$$

is the total spin. D-theory is defined by the quantum partition function

$Z=\operatorname{Tr} \exp (-\beta H)$.

The trace is taken in the Hilbert space, which is a direct product of the Hilbert spaces of individual spins. It should be noted that D-theory can be formulated with any value of the spin, not only with spin $1 / 2$.

At this point, we have replaced the 2-d $O(3)$ model, formulated in terms of classical fields $\vec{s}_{x}$, by a 2 -d system of quantum spins $\vec{S}_{x}$ with the same symmetries. The inverse temperature $\beta$ of the quantum system can be viewed as the extent of an additional third dimension. In the standard interpretation of the 2-d quantum spin system this dimension would be Euclidean time. In D-theory, however, Euclidean time is already part of the 2-d lattice. Indeed, as we will see, the additional Euclidean dimension ultimately disappears via dimensional reduction. The 2 -d antiferromagnetic spin $1 / 2$ quantum Heisenberg model has very interesting properties. It describes the undoped precursor insulators of high-temperature superconductors - materials like $\mathrm{La}_{2} \mathrm{CuO}_{4}-$ whose ground states are Néel ordered with a spontaneously generated staggered magnetization. Indeed, there is overwhelming numerical evidence that the ground state of the 2-d antiferromagnetic spin $1 / 2$ quantum Heisenberg model exhibits long-range order [7 9]. The same is true for higher spins, and thus the following discussion applies equally well to all spin values. In practice, however, the smallest spin $1 / 2$ is most interesting, because it allows us to represent the physics of the 2-d $O(3)$ model in the smallest possible Hilbert space.

Formulating the 2-d quantum model as a path integral in the extra dimension results in a $3-\mathrm{d}$ $S O(3)$-symmetric classical model. At zero temperature of the quantum system we are in the infinite volume limit of the corresponding $3-\mathrm{d}$ model. The Néel order of the ground state of the 2-d quantum system implies that the corresponding 3-d classical system is in a broken phase, in which only an $S O(2)$ symmetry remains intact. As a consequence of Goldstone's theorem, two massless bosons arise - in this case two antiferromagnetic magnons (or spin-waves). Using chiral perturbation theory one can describe the magnon dynamics at low energies [10]. The Goldstone bosons are represented by fields in the coset $S O(3) / S O(2)=S^{2}$, which resembles a 2dimensional sphere. Consequently, the magnons are described by 3 -component unit vectors $\vec{s}-$ the same fields that appear in the original 2-d $O(3)$ model. Indeed, due to spontaneous symmetry breaking, the collective excitations of many discrete quantum spin variables form an effective continuous classical field $\vec{s}$. This is one of the main dynamical ingredients of D-theory.

Another ingredient is dimensional reduction, to which we now turn. To lowest order in chiral perturbation theory, the effective action of the Goldstone bosons takes the form

$S[\vec{s}]=\int_{0}^{\beta} d x_{3} \int d^{2} x \frac{\rho_{s}}{2}\left[\partial_{\mu} \vec{s} \cdot \partial_{\mu} \vec{s}+\frac{1}{c^{2}} \partial_{3} \vec{s} \cdot \partial_{3} \vec{s}\right] \cdot(7)$

Here $c$ and $\rho_{s}$ are the spin-wave velocity and the spin stiffness. Note that $\mu$ extends over the physical space-time indices 1 and 2 only. The 2 -d quantum system at finite temperature corresponds to a 3 -d classical model with finite extent $\beta$ in the extra dimension. For massless particles - i.e., in the presence of an infinite correlation length $\xi$ - the finite temperature system appears dimensionally reduced to two dimensions, because $\beta \ll \xi$. In two dimensions, however, the MerminWagner-Coleman theorem prevents the existence of interacting massless Goldstone bosons [11, and, indeed, the 2-d $O(3)$ model has a nonperturbatively generated mass-gap. Hasenfratz and Niedermayer suggested to use a block spin renormalization group transformation to map the 3 -d $O(3)$ model with finite extent $\beta$ to a 2 -d lattice $O(3)$ model [12]. One averages the 3 -d field over volumes of size $\beta$ in the third direction and $\beta c$ in the two space-time directions. Due to the large correlation length, the field is essentially constant over these blocks. The averaged field is defined 
at the block centers, which form a 2-d lattice of spacing $\beta c$. Note that this lattice spacing is different from the lattice spacing of the original quantum antiferromagnet. The effective action of the averaged field defines a 2-d lattice $O(3)$ model, formulated in Wilson's framework. Using chiral perturbation theory, Hasenfratz and Niedermayer expressed its coupling constant as

$1 / g=\beta \rho_{s}+\mathcal{O}\left(1 / \beta \rho_{s}\right)$.

Using the 3-loop $\beta$-function of the 2-d $O(3)$ model together with its exact mass-gap [13], they also extended an earlier result of Chakravarty, Halperin and Nelson 14 for the inverse correlation length of the quantum antiferromagnet to

$\xi=\frac{e c}{16 \pi \rho_{s}} \exp \left(2 \pi \beta \rho_{s}\right)\left[1-\frac{1}{4 \pi \beta \rho_{s}}+\mathcal{O}\left(1 / \beta^{2} \rho_{s}^{2}\right)\right]$.

Here $e$ is the base of the natural logarithm. The above equation resembles the asymptotic scaling behavior of the 2-d classical $O(3)$ model. In fact, one can view the 2-d antiferromagnetic quantum Heisenberg model in the zero temperature limit as a regularization of the $2-\mathrm{d} O(3)$ model. It is remarkable that this D-theory formulation is entirely discrete, even though the model is usually formulated with a continuous classical configuration space.

As illustrated in figure 1, the dimensionally reduced effective 2-d theory is a Wilsonian lattice theory with lattice spacing $\beta c$. The continuum limit of that theory is reached as $g=1 / \beta \rho_{s} \rightarrow 0$, and hence as the extent $\beta$ of the extra dimension becomes large. Still, in physical units of the correlation length, the extent $\beta \ll \xi$ becomes negligible in this limit, and hence the theory undergoes dimensional reduction to two dimensions. In the continuum limit, the lattice spacing $\beta c$ of the effective 2-d Wilsonian lattice $O(3)$ model becomes large in units of the microscopic lattice spacing of the quantum spin system. Therefore, D-theory introduces a discrete substructure underlying Wilson's lattice theory. This substructure is defined on a very fine microscopic lattice. In other words, D-theory regularizes quantum fields at much shorter distance scales than the ones considered in Wilson's formulation. For practical purposes it is essential that the effective

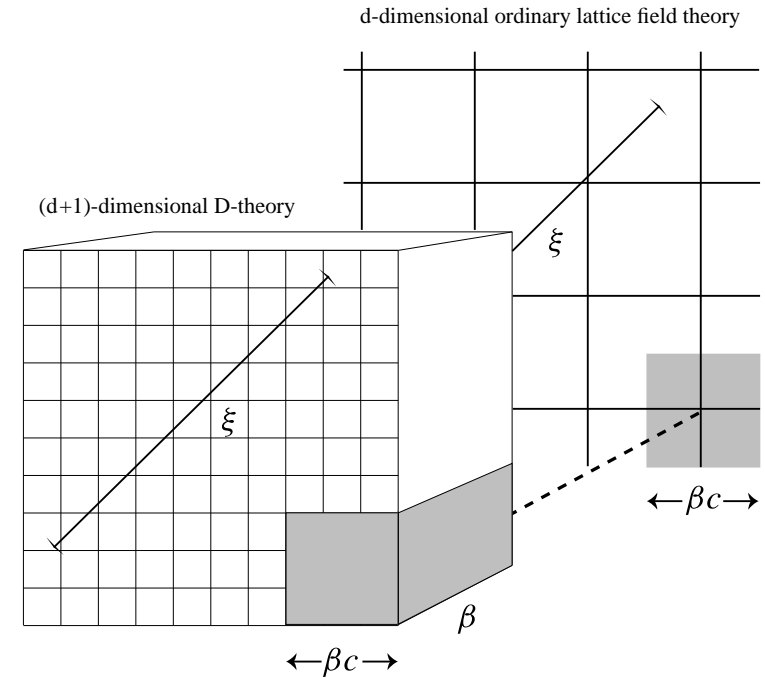

Figure 1. Dimensional reduction of a D-theory: Averaging the $(d+1)$-dimensional effective field of the D-theory over blocks of size $\beta$ in the extra dimension and $\beta c$ in the physical directions results in an effective $d$-dimensional Wilsonian lattice field theory with lattice spacing $\beta c$.

Wilsonian lattice gauge theory results from exact blocking of the continuum field theory describing the low-energy excitations of the underlying Dtheory. Hence, the resulting Wilsonian lattice action is perfect, up to cut-off effects due to the microscopic D-theory lattice, such that in D-theory simulations lattices need not be finer than in the standard approach.

The additional microscopic structure may provide new insight into the long-distance continuum physics. In the context of the quantum Heisenberg model, the microscopic substructure is due to the presence of electrons hopping on a crystal lattice. After all, the spin-waves of a quantum antiferromagnet are just collective excitations of the spins of many electrons. In the same way, gluons appear as collective excitations of rishons hopping on the microscopic lattice of the corresponding quantum link model for QCD. In that case, the lattice is most likely unphysical, because it just serves as a regulator. However, even if the rishons propagate only at the cut-off scale, they may still be useful for understanding the physics 
in the continuum limit. Let us illustrate the rishon ideas in the context of the quantum Heisenberg model. Then the rishons can be identified with physical electrons. In fact, the quantum spin operator at a lattice site $x$,

$\vec{S}_{x}=\frac{1}{2} \sum_{i, j} c_{x}^{i \dagger} \vec{\sigma}_{i j} c_{x}^{j}$,

can be expressed in terms of Pauli matrices $\vec{\sigma}$ and electron creation and annihilation operators $c_{x}^{i \dagger}$ and $c_{x}^{i}(i, j \in\{1,2\})$ with the usual anticommutation relations

$$
\left\{c_{x}^{i \dagger}, c_{y}^{j \dagger}\right\}=\left\{c_{x}^{j}, c_{y}^{j}\right\}=0, \quad\left\{c_{x}^{i}, c_{y}^{j \dagger}\right\}=\delta_{x, y} \delta_{i j} .
$$

It is straightforward to show that $\vec{S}_{x}$, constructed in this way, has the correct commutation relations. In fact, the commutation relations are also satisfied when the rishons are quantized as bosons. The rishon representation allows us to rewrite the Hamilton operator in terms of singlet combinations $\sum_{i} c_{x}^{i \dagger} c_{y}^{i}$, with interesting consequences for $O(N)$ quantum spin models in the large $N$ limit. It should be noted that the total number of rishons at each site $x$ is a conserved quantity, because the local rishon number operator $\mathcal{N}_{x}=\sum_{i} c_{x}^{i \dagger} c_{x}^{i}$ commutes with the Hamiltonian. In fact, fixing the number of rishons is equivalent to selecting a value for the spin, i.e., to choosing an irreducible representation.

The discrete nature of the D-theory degrees of freedom allows the application of very efficient cluster algorithms. The quantum Heisenberg model, for example, can be treated with a loop cluster algorithm 15, 8]. Defining the path integral for discrete quantum systems does not even require discretization of the additional Euclidean dimension. This observation has led to a very efficient loop cluster algorithm operating directly in the continuum of the extra dimension [9. This algorithm, combined with a finite-size scaling technique, has been used to study the correlation length of the Heisenberg model up to $\xi \approx 350000$ lattice spacings [16]. In this way the analytic prediction of Hasenfratz and Niedermayer - and hence the scenario of dimensional reduction - has been verified numerically. This shows explicitly that the 2-d $\mathrm{O}(3)$ model can be investigated very efficiently using D-theory, i.e., by simulating the $(2+1)$-d path integral for the 2 -d quantum Heisenberg model. In this case, the numerical effort is compatible to simulating the 2d $O(3)$ model directly with the Wolff cluster algorithm 17]. However, for most other lattice models - for example, for gauge theories - despite numerous attempts, no efficient cluster algorithm has been found in Wilson's formulation. If an efficient cluster algorithm can be constructed for the D-theory formulation, it would allow simulations more accurate than the ones presently possible. Indeed, recently, the first cluster algorithm for a model with a continuous gauge group has been constructed for a $U(1)$ quantum link model [5], and it is plausible that it can be generalized to non-Abelian gauge groups.

The exponential divergence of the correlation length is due to the asymptotic freedom of the 2d $O(3)$ model. Hence, one might expect that the above scenario of dimensional reduction is specific to $d=2$. As we will see now, dimensional reduction also occurs in higher dimensions, but in a slightly different way. Let us consider the antiferromagnetic quantum Heisenberg model on a $d$-dimensional lattice with $d>2$. Then, again, the ground state has a broken symmetry, and the low energy excitations of the system are two massless magnons. The effective action of chiral perturbation theory is the same as before, except that the integration now extends over a higher-dimensional space. Again, at an infinite extent $\beta$ of the extra dimension, one has an infinite correlation length. Thus, once $\beta$ becomes finite, the extent of the extra dimension is negligible compared to the correlation length, and the theory undergoes dimensional reduction to $d$ dimensions. However, in contrast to the $d=2$ case, now there is no reason why the Goldstone bosons should pick up a mass after dimensional reduction. Consequently, the correlation length remains infinite, and we end up in a $d$-dimensional phase with broken symmetry. When the extent $\beta$ is reduced further, we eventually reach the symmetric phase, in which the correlation length is finite. The transition between the broken and symmetric phase is known to be of second order. Thus, approaching the phase transition from the 
symmetric phase at low $\beta$ also leads to a divergent correlation length, and hence, again, to dimensional reduction. Thus, the universal continuum physics of $O(3)$ models in any dimension $d \geq 2$ is naturally contained in the framework of D-theory.

Still, the $d=1$ case requires a separate discussion. The behavior of quantum spin chains depends crucially on the value of the spin. Haldane has conjectured that 1-d antiferromagnetic $O(3)$ quantum spin chains with integer spins have a mass-gap, while those with half-integer spins are gapless [18]. This conjecture is by now verified in great detail. For example, the spin 1/2 antiferromagnetic Heisenberg chain has been solved by the Bethe ansatz, and indeed turns out to have no mass-gap 19]. The same has been shown analytically for all half-integer spins 20]. On the other hand, there is strong numerical evidence for a mass-gap in spin 1 and spin 2 systems 21]. Hence, only for half-integer spins the $(1+1)$-dimensional D-theory with an infinite extent $\beta$ in the second direction has an infinite correlation length. The low-energy effective theory for this system is the 2 -d $O(3)$ model at vacuum angle $\theta=\pi$, i.e.,

$$
\begin{aligned}
S[\vec{s}] & =\int_{0}^{\beta} d x_{1} \int d x_{2}\left\{\frac{1}{2 g^{2}}\left[\partial_{1} \vec{s} \cdot \partial_{1} \vec{s}+\frac{1}{c^{2}} \partial_{2} \vec{s} \cdot \partial_{2} \vec{s}\right]\right. \\
& \left.+\frac{i \theta}{4 \pi} \vec{s} \cdot\left[\partial_{1} \vec{s} \times \partial_{2} \vec{s}\right]\right\},
\end{aligned}
$$

as conjectured by Haldane [18]. This model is in the universality class of a 2 -d conformal field theory — the $k=1$ Wess-Zumino-Novikov-Witten model - as was first argued by Affleck [22]. Indeed, it has been shown numerically that the mass-gap of the 2-d $O(3)$ model (which is present at $\theta \neq \pi$ ) disappears at $\theta=\pi$ [23]. The simulation of the 2-d $O(3)$ model at $\theta=\pi$ is extremely difficult due to the complex action. Still, it is feasible using the Wolff cluster algorithm combined with an appropriate improved estimator for the topological charge distribution. It is remarkable that no complex action arises in the D-theory formulation of this problem, and the simulation is hence much simpler.

So far, we have seen that D-theory naturally contains the continuum physics of $O(3)$ models. This alone would be interesting. However, as we will see, D-theory is far more general. It can be extended to other scalar field theories and to gauge theories with various symmetries and in various space-time dimensions.

\section{D-Theory Representation of Complex Vectors and Matrices}

As we have seen, in the low-temperature limit the 2 -d spin $1 / 2$ quantum Heisenberg model provides a D-theory regularization for the 2-d $O(3)$ symmetric continuum field theory. In that case, a vector of Pauli matrices replaces the 3-component unit-vector of a classical field configuration. Here, this structure is generalized to other fields.

\subsection{Complex Vectors}

In $C P(N-1)$ models, classical $N$-component complex vectors $z$ arise. We will now discuss their representation in D-theory. The symmetry group of a $C P(N-1)$ model is $U(N)$, which has $N^{2}$ generators. In D-theory the complex components $z^{i}$ are represented by $2 N$ Hermitean operators $N$ for the real and $N$ for the imaginary parts. Hence, the total number of generators is $N^{2}+$ $2 N=(N+1)^{2}-1$ - the number of generators of $S U(N+1)$. A rishon representation of the $S U(N+1)$ algebra is given by

$Z^{i}=c^{0 \dagger} c^{i}, \vec{G}=\sum_{i j} c^{i \dagger} \vec{\lambda}_{i j} c^{j}, G=\sum_{i} c^{i \dagger} c^{i}$.

Here $\vec{\lambda}$ is the vector of generators for $S U(N)$, which obeys $\left[\lambda^{a}, \lambda^{b}\right]=2 i f_{a b c} \lambda^{c}$, as well as $\operatorname{Tr}\left(\lambda^{a} \lambda^{b}\right)=2 \delta_{a b}$. The quantum operator $Z^{i}$ replaces the classical variable $z^{i}, \vec{G}$ is a vector of $S U(N)$ generators obeying $\left[G^{a}, G^{b}\right]=2 i f_{a b c} G^{c}$, and $G$ is a $U(1)$ generator.

\subsection{Complex Matrices}

$U(N)$ gauge theories are formulated in terms of classical complex matrix link variables. The corresponding $S U(N)_{L} \otimes S U(N)_{R} \otimes U(1)$ gauge transformations at the left- and right-hand side of a link are generated by $2\left(N^{2}-1\right)+1$ Hermitean operators. In D-theory a classical complex valued matrix $u$ is replaced by a matrix $U$, whose elements are non-commuting operators. The elements of the matrix $U$ are described by $2 N^{2}$ Hermitean generators $-N^{2}$ representing the real 
part and $N^{2}$ representing the imaginary part of the classical matrix $u$. Altogether, we thus have $2\left(N^{2}-1\right)+1+2 N^{2}=4 N^{2}-1$ generators the number of generators of $S U(2 N)$. A rishon representation of the $S U(2 N)$ algebra is given by

$$
\begin{aligned}
& U^{i j}=c_{+}^{i \dagger} c_{-}^{j}, \\
& \vec{L}=\sum_{i j} c_{+}^{i \dagger} \vec{\lambda}_{i j} c_{+}^{j}, \vec{R}=\sum_{i j} c_{-}^{i \dagger} \vec{\lambda}_{i j} c_{-}^{j}, \\
& T=\sum_{i}\left(c_{+}^{i \dagger} c_{+}^{i}-c_{-}^{i \dagger} c_{-}^{i}\right) .
\end{aligned}
$$

There are two sets of rishons, $c_{+}^{i}$ and $c_{-}^{i}$, associated with the left and right $S U(N)$ symmetries generated by $\vec{L}$ and $\vec{R}$, and $T$ is a $U(1)$ generator.

To summarize, in D-theory classical complex vectors $z$ are replaced by vectors of operators $Z$, which are embedded in an $S U(N+1)$ algebra, and classical complex matrices $u$ are replaced by matrices $U$ with operator valued elements, which are embedded in the algebra of $S U(2 N)$. Similarly, real valued classical vectors and matrices can be replaced by corresponding operator valued objects embedded in the algebras of $S O(N+1)$ and $S O(2 N)$, respectively. In the next section we will use these basic structures to construct some D-theory models, both with global and local symmetries.

\section{D-theory Formulation of Specific Mod- els}

In this section some quantum field theories are formulated in the framework of D-theory. Models with global and local symmetries are constructed explicitly. The Hamilton operator $H$ of a model is defined on a $d$-dimensional lattice. It replaces the Euclidean action in the standard formulation of lattice field theory. In D-theory, the partition function takes the quantum form $Z=\operatorname{Tr} \exp (-\beta H)$. The dynamics of the models - in particular, their dimensional reduction will be discussed in section 5 .

\section{1. $U(N)$ and $S U(N)$ Quantum Link Mod- els}

Wilson's formulation of lattice gauge theory uses classical complex $S U(N)$ parallel transporter link matrices $u_{x, \mu}$, with an action

$$
S[u]=-\sum_{x, \mu \neq \nu} \operatorname{Tr}\left[u_{x, \mu} u_{x+\hat{\mu}, \nu} u_{x+\hat{\nu}, \mu}^{\dagger} u_{x, \nu}^{\dagger}\right]
$$

The action is invariant under $S U(N)$ gauge transformations

$u_{x, \mu}^{\prime}=\exp \left(i \vec{\alpha}_{x} \cdot \vec{\lambda}\right) u_{x, \mu} \exp \left(-i \vec{\alpha}_{x+\hat{\mu}} \cdot \vec{\lambda}\right)$.

In D-theory the action is replaced by the Hamilton operator

$H=J \sum_{x, \mu \neq \nu} \operatorname{Tr}\left[U_{x, \mu} U_{x+\hat{\mu}, \nu} U_{x+\hat{\nu}, \mu}^{\dagger} U_{x, \nu}^{\dagger}\right]$.

Here the elements of the $N \times N$ quantum link operators $U_{x, \mu}$ consist of generators of $S U(2 N)$. Gauge invariance now means that $H$ commutes with the local generators $\vec{G}_{x}$ of gauge transformations at the site $x$, which obey

$\left[G_{x}^{a}, G_{y}^{b}\right]=2 i \delta_{x y} f_{a b c} G_{x}^{c}$.

Gauge covariance of a quantum link variable requires

$$
\begin{aligned}
U_{x, \mu}^{\prime} & =\prod_{y} \exp \left(-i \vec{\alpha}_{y} \cdot \vec{G}_{y}\right) U_{x, \mu} \prod_{z} \exp \left(i \vec{\alpha}_{z} \cdot \vec{G}_{z}\right) \\
& =\exp \left(i \vec{\alpha}_{x} \cdot \vec{\lambda}\right) U_{x, \mu} \exp \left(-i \vec{\alpha}_{x+\hat{\mu}} \cdot \vec{\lambda}\right)
\end{aligned}
$$

where $\prod_{x} \exp \left(i \vec{\alpha}_{x} \cdot \vec{G}_{x}\right)$ is the unitary operator that represents a general gauge transformation in Hilbert space. The above equation implies the following commutation relation

$\left[\vec{G}_{x}, U_{y, \mu}\right]=\delta_{x, y+\hat{\mu}} U_{y, \mu} \vec{\lambda}-\delta_{x, y} \vec{\lambda} U_{y, \mu}$.

It is straightforward to show that this is satisfied when we write

$\vec{G}_{x}=\sum_{\mu}\left(\vec{R}_{x-\hat{\mu}, \mu}+\vec{L}_{x, \mu}\right)$,

where $\vec{R}_{x, \mu}$ and $\vec{L}_{x, \mu}$ are generators of right and left gauge transformations of the link variable $U_{x, \mu}$. The commutation relations of eq.(20) imply

$$
\begin{aligned}
{\left[\vec{R}_{x, \mu}, U_{y, \nu}\right] } & =\delta_{x, y} \delta_{\mu \nu} U_{x, \mu} \vec{\lambda}, \\
{\left[\vec{L}_{x, \mu}, U_{y, \nu}\right] } & =-\delta_{x, y} \delta_{\mu \nu} \vec{\lambda} U_{x, \mu} .
\end{aligned}
$$


The rishon representation is given by

$U_{x, \mu}^{i j}=c_{x,+\mu}^{i \dagger} c_{x+\hat{\mu},-\mu}^{j}$,

$\vec{L}_{x, \mu}=\sum_{i j} c_{x,+\mu}^{i \dagger} \vec{\lambda}_{i j} c_{x,+\mu}^{j}$,

$\vec{R}_{x, \mu}=\sum_{i j} c_{x+\hat{\mu},-\mu}^{i \dagger} \vec{\lambda}_{i j} c_{x+\hat{\mu},-\mu}^{j}$.

The rishon operators obey canonical anticommutation relations

$\left\{c_{x, \pm \mu}^{i}, c_{y, \pm \nu}^{j \dagger}\right\}=\delta_{x y} \delta_{ \pm \mu, \pm \nu} \delta_{i j}$,

$\left\{c_{x, \pm \mu}^{i}, c_{y, \pm \nu}^{j}\right\}=0,\left\{c_{x, \pm \mu}^{i \dagger}, c_{y, \pm \nu}^{j \dagger}\right\}=0$.

The whole algebra commutes with the rishon number operator

$\mathcal{N}_{x, \mu}=\sum_{i}\left(c_{x,+\mu}^{i \dagger} c_{x,+\mu}^{i}+c_{x+\hat{\mu},-\mu}^{i \dagger} c_{x+\hat{\mu},-\mu}^{i}\right)$

on each individual link. Together with the generator

$T_{x, \mu}=\sum_{i}\left(c_{x,+\mu}^{i \dagger} c_{x,+\mu}^{i}-c_{x+\hat{\mu},-\mu}^{i \dagger} c_{x+\hat{\mu},-\mu}^{i}\right)$.

the above operators form the link based algebra of $S U(2 N)$. One finds

$\left[T_{x, \mu}, U_{y, \nu}\right]=2 \delta_{x, y} \delta_{\mu \nu} U_{x, \mu}$,

which implies that

$G_{x}=\frac{1}{2} \sum_{\mu}\left(T_{x-\hat{\mu}, \mu}-T_{x, \mu}\right)$

generates an additional $U(1)$ gauge transformation, i.e.

$$
\begin{aligned}
U_{x, \mu}^{\prime} & =\prod_{y} \exp \left(-i \alpha_{y} G_{y}\right) U_{x, \mu} \prod_{z} \exp \left(i \alpha_{z} G_{z}\right) \\
& =\exp \left(i \alpha_{x}\right) U_{x, \mu} \exp \left(-i \alpha_{x+\mu}\right) .
\end{aligned}
$$

Indeed the Hamilton operator of eq.(17) is also invariant under the extra $U(1)$ gauge transformations and thus describes a $U(N)$ lattice gauge theory.

To reduce the symmetry of the quantum link model from $U(N)$ to $S U(N)$ one breaks the additional $U(1)$ gauge symmetry by adding the real
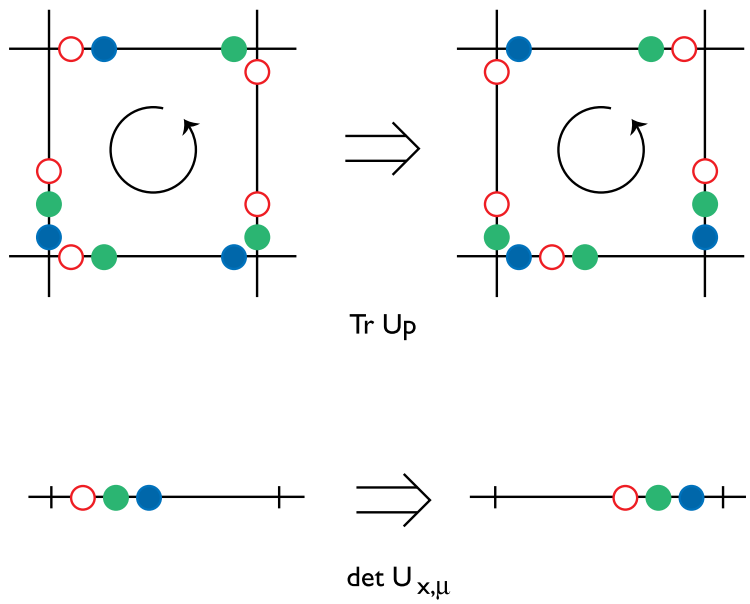

Figure 2. QCD rishon dynamics: The trace part of the Hamiltonian induces hopping of rishons of various colors around a plaquette. The determinant part shifts a color-neutral combination of $N$ rishons from one end of a link to the other.

part of the determinant of each link matrix to the Hamilton operator

$$
\begin{aligned}
H & =J \sum_{x, \mu \neq \nu} \operatorname{Tr}\left[U_{x, \mu} U_{x+\hat{\mu}, \nu} U_{x+\hat{\nu}, \mu}^{\dagger} U_{x, \nu}^{\dagger}\right] \\
& +J^{\prime} \sum_{x, \mu}\left[\operatorname{det} U_{x, \mu}+\operatorname{det} U_{x, \mu}^{\dagger}\right] .
\end{aligned}
$$

The $U(N)$ symmetry can be reduced to $S U(N)$ via the determinant, only when one works with $\mathcal{N}_{x, \mu}=N$ rishons on each link. This corresponds to choosing the $(2 N) ! /(N !)^{2}$-dimensional representation of $S U(2 N)$. The rishon dynamics is illustrated in figure 2 .

\subsection{Quantum $C P(N-1)$ Models}

$C P(N-1)$ models are interesting, because they have a global $S U(N)$ symmetry as well as a $U(1)$ gauge invariance. However, the gauge fields are just auxiliary fields in this case. In the standard formulation of lattice field theory $C P(N-1)$ models are formulated in terms of classical complex unit-vectors $z_{x}$ and complex link variables $u_{x, \mu}$. The action can be written as

$$
S[z, u]=-\sum_{x, \mu}\left(z_{x}^{\dagger} u_{x, \mu} z_{x+\hat{\mu}}+z_{x+\hat{\mu}}^{\dagger} u_{x, \mu}^{\dagger} z_{x}\right) .
$$


Note that there is no plaquette term for the gauge field. Consequently, the gauge field can be integrated out - it only acts as an auxiliary field. In D-theory the corresponding Hamilton operator takes the form

$H=J \sum_{x, \mu}\left(Z_{x}^{\dagger} U_{x, \mu} Z_{x+\hat{\mu}}+Z_{x+\hat{\mu}}^{\dagger} U_{x, \mu}^{\dagger} Z_{x}\right)$.

Here the $N$ components $Z_{x}^{i}$ of the quantum spin $Z_{x}$ consist of $2 N$ Hermitean generators of $S U(N+1)$, and $U_{x, \mu}=S_{x, \mu}^{1}+i S_{x, \mu}^{2}=S_{x, \mu}^{+}$is the raising operator of an $S U(2)$ algebra on each link. In rishon representation we have

$Z_{x}^{i}=c_{x}^{0 \dagger} c_{x}^{i}, U_{x, \mu}=c_{x,+\mu}^{\dagger} c_{x+\hat{\mu},-\mu}$.

Global $S U(N)$ transformations are generated by

$\vec{G}=\sum_{x} \sum_{i j} c_{x}^{i \dagger} \vec{\lambda}_{i j} c_{x}^{j}$

and the generator of $U(1)$ gauge transformations takes the form

$G_{x}=\frac{1}{2} \sum_{\mu}\left(T_{x-\hat{\mu}, \mu}-T_{x, \mu}\right)+\sum_{i} c_{x}^{i \dagger} c_{x}^{i}$.

In rishon representation we have

$T_{x, \mu}=c_{x,+\mu}^{\dagger} c_{x,+\mu}-c_{x+\hat{\mu},-\mu}^{\dagger} c_{x+\hat{\mu},-\mu}$.

The invariance properties of the model follow from $[H, \vec{G}]=\left[H, G_{x}\right]=0$. In this case, the rishon numbers on each site and on each link are separately conserved.

It should be clear by now that D-theory is a very general structure, which naturally provides us with lattice field theories formulated in terms of discrete variables - quantum spins and quantum links. The cases worked out here in some detail are examples that demonstrate the generality of D-theory. There are certainly more models one could investigate. Next, we want to argue that D-theory does not define a new set of field theories. It just provides a new non-perturbative regularization and quantization of the corresponding classical models. To understand this, we must address the issue of dimensional reduction.

\section{Classical Gauge Fields from Dimen- sional Reduction of Quantum Links}

As we have seen in detail in section 2 , the quantum Heisenberg model provides a D-theory regularization for the 2-d $O(3)$ model. The connection between the two models is established using chiral perturbation theory. The Goldstone boson fields $\vec{s}$ describing the low-energy dynamics of the Heisenberg model emerge as collective excitations of the quantum spins. By dimensional reduction they become the effective 2-d fields of a lattice $O(3)$ model. The continuum limit of this lattice model is reached when the extent $\beta$ of the extra Euclidean dimension becomes large. The success of D-theory relies entirely on the fact that the $(d+1)$-dimensional theory is massless, i.e., that in the ground state of the quantum Heisenberg model the $S O(3)$ symmetry is spontaneously broken to $S O(2)$. Only then chiral perturbation theory applies, and dimensional reduction occurs. The same is true for other D-theory quantum spin models.

Let us now consider $S U(N)$ non-Abelian gauge theories in $d=4$. The Hamilton operator of the corresponding quantum link model, which is defined on a 4-d lattice, describes the evolution of the system in a fifth Euclidean direction. The partition function $Z=\operatorname{Tr} \exp (-\beta H)$ can then be represented as a $(4+1)$-d path integral. Note that we have not included a projector on gauge invariant states, i.e., gauge variant states also propagate in the fifth direction. This means that we do not impose a Gauss law in the unphysical direction. Not imposing Gauss' law implies $A_{5}=0$ for the fifth component of the gauge potential. This is important, because it leaves us with the correct field content after dimensional reduction. Of course, the physical Gauss law is properly imposed, because the model does contain non-trivial Polyakov loops in the Euclidean time direction.

Dimensional reduction in quantum link models works differently than for quantum spins. In the spin case the spontaneous breakdown of a global symmetry provides the massless Goldstone modes that are necessary for dimensional reduction. On the other hand, when a gauge symmetry breaks spontaneously, the Higgs mechanism gives mass 
to the gauge bosons, and dimensional reduction would not occur. Fortunately, non-Abelian gauge theories in five dimensions are generically in a massless Coulomb phase 24]. This has recently been verified in detail for 5-d $S U(2)$ and $S U(3)$ lattice gauge theories using Wilson's formulation [5]. Here, we assume that the same is true for quantum link models - an assumption that can only be checked in numerical simulations. The leading terms in the low-energy effective action of 5 -d Coulombic gluons take the form

$$
\begin{aligned}
S[A] & =\int_{0}^{\beta} d x_{5} \int d^{4} x \frac{1}{2 e^{2}}\left[\operatorname{Tr} F_{\mu \nu} F_{\mu \nu}\right. \\
& \left.+\frac{1}{c^{2}} \operatorname{Tr} \partial_{5} A_{\mu} \partial_{5} A_{\mu}\right] .
\end{aligned}
$$

The quantum link model leads to a 5 -d gauge theory characterized by the "velocity of light" $c$. Note that here $\mu$ runs over 4 -d indices only. The dimensionful 5 -d gauge coupling $1 / e^{2}$ is the analog of $\rho_{s}$ in the spin models. At finite $\beta$ the above theory has only a 4 -d gauge invariance, because we have fixed $A_{5}=0$, i.e., we have not imposed the Gauss law. At $\beta=\infty$ we are in the 5 -d Coulomb phase with massless gluons, and thus with an infinite correlation length $\xi$. When $\beta$ is made finite, the extent of the extra dimension is negligible compared to $\xi$. Hence, the theory appears to be dimensionally reduced to four dimensions. Of course, in four dimensions the confinement hypothesis suggests that gluons are no longer massless. Indeed, as it was argued in ref.[3], a finite correlation length

$\xi \propto \exp \left(\frac{24 \pi^{2} \beta}{11 N e^{2}}\right)$

is expected to be generated non-perturbatively. Here $24 \pi^{2} / 11 N$ is the 1 -loop $\beta$-function coefficient of $S U(N)$ gauge theory. In contrast to the spin models, the exact value for the mass-gap (and hence the prefactor of the exponential) is not known in this case. For large $\beta$ the gauge coupling of the dimensionally reduced 4-d theory is given by

$1 / g^{2}=\beta / e^{2}$.

Thus the continuum limit $g \rightarrow 0$ of the 4 -d theory is approached when one sends the extent $\beta$ of the fifth direction to infinity. Hence, dimensional reduction occurs when the extent of the fifth direction becomes large. This is due to asymptotic freedom, which implies that the correlation length grows exponentially with $\beta$. As in the spin models, it is useful to think of the dimensionally reduced 4-d theory as a Wilsonian lattice theory with lattice spacing $\beta c$ (which has nothing to do with the lattice spacing of the quantum link model). In fact, one can again imagine performing a block renormalization group transformation that averages the 5 -d field over cubic blocks of size $\beta$ in the fifth direction and of size $\beta c$ in the four physical space-time directions. The block centers then form a 4-d space-time lattice of spacing $\beta c$ and the effective theory of the block averaged $5-\mathrm{d}$ field is indeed an ordinary 4-d lattice theory.

The situation for 4-d $U(1)$ gauge theory is different. In contrast to non-Abelian gauge theories, after dimensional reduction from five to four dimensions, there is no reason for the photons to pick up a mass. They can exist in a 4-d Coulomb phase, because they are not confined. Hence, dimensional reduction occurs already at a finite extent of the fifth dimension - not only in the $\beta \rightarrow \infty$ limit. Still, when $\beta$ becomes too small, one enters the strong coupling confined phase, which has a finite correlation length. If the phase transition between the confined phase and the Coulomb phase is of second order, one obtains universal continuum behavior via dimensional reduction.

\section{Inclusion of Fermion Fields}

In order to formulate realistic quantum field theories in the framework of D-theory, it is important to include fermion fields. Even in Wilson's formulation of lattice field theory, fermions already live in a finite Hilbert space per site. Thus, their treatment can be taken over to D-theory with almost no change. Here, we illustrate this for QCD. Other models with fermions can be constructed along the same lines. In lattice gauge theory the fermion doubling problem arises. Wilson solved that problem by breaking chiral symmetry explicitly. The standard Wilson action for 
$\mathrm{QCD}$, for example, is given by

$$
\begin{aligned}
& S[\bar{\psi}, \psi, u]=-\sum_{x, \mu \neq \nu} \operatorname{Tr}\left[u_{x, \mu} u_{x+\hat{\mu}, \nu} u_{x+\hat{\nu}, \mu}^{\dagger} u_{x, \nu}^{\dagger}\right] \\
& +\frac{1}{2} \sum_{x, \mu}\left[\bar{\psi}_{x} \gamma_{\mu} u_{x, \mu} \psi_{x+\hat{\mu}}-\bar{\psi}_{x+\hat{\mu}} \gamma_{\mu} u_{x, \mu}^{\dagger} \psi_{x}\right] \\
& +M \sum_{x} \bar{\psi}_{x} \psi_{x}+\frac{r}{2} \sum_{x, \mu}\left[2 \bar{\psi}_{x} \psi_{x}\right. \\
& \left.-\bar{\psi}_{x} u_{x, \mu} \psi_{x+\hat{\mu}}-\bar{\psi}_{x+\hat{\mu}} u_{x, \mu}^{\dagger} \psi_{x}\right] .
\end{aligned}
$$

Here $\bar{\psi}_{x}$ and $\psi_{x}$ are independent Grassmann valued spinors associated with the lattice site $x, \gamma_{\mu}$ are Dirac matrices and $M$ is the bare quark mass. The Wilson term (proportional to $r$ ) removes the doubler fermions, but also breaks chiral symmetry. In practice, this leads to several problems, because $M$ must be tuned appropriately in order to reach the chiral limit.

The construction principle of D-theory is to replace the classical action in Wilson's formulation by a Hamilton operator that describes the evolution of the system in an additional Euclidean direction. For fermions one must also replace $\bar{\psi}_{x}$ by $\Psi_{x}^{\dagger} \gamma_{5}$. Hence, in D-theory, QCD is described by the Hamilton operator

$$
\begin{aligned}
H & =J \sum_{x, \mu \neq \nu} \operatorname{Tr}\left[U_{x, \mu} U_{x+\hat{\mu}, \nu} U_{x+\hat{\nu}, \mu}^{\dagger} U_{x, \nu}^{\dagger}\right] \\
& +J^{\prime} \sum_{x, \mu}\left[\operatorname{det} U_{x, \mu}+\operatorname{det} U_{x, \mu}^{\dagger}\right] \\
& +\frac{1}{2} \sum_{x, \mu}\left[\Psi_{x}^{\dagger} \gamma_{5} \gamma_{\mu} U_{x, \mu} \Psi_{x+\hat{\mu}}-\Psi_{x+\hat{\mu}}^{\dagger} \gamma_{5} \gamma_{\mu} U_{x, \mu}^{\dagger} \Psi_{x}\right] \\
& +M \sum_{x} \Psi_{x}^{\dagger} \gamma_{5} \Psi_{x}+\frac{r}{2} \sum_{x, \mu}\left[2 \Psi_{x}^{\dagger} \gamma_{5} \Psi_{x}\right. \\
& \left.-\Psi_{x}^{\dagger} \gamma_{5} U_{x, \mu} \Psi_{x+\hat{\mu}}-\Psi_{x+\hat{\mu}}^{\dagger} \gamma_{5} U_{x, \mu}^{\dagger} \Psi_{x}\right] .
\end{aligned}
$$

Here $\Psi_{x}^{\dagger}$ and $\Psi_{x}$ are quark creation and annihilation operators with canonical anti-commutation relations

$$
\begin{aligned}
& \left\{\Psi_{x}^{i a \alpha}, \Psi_{y}^{j b \beta \dagger}\right\}=\delta_{x y} \delta_{i j} \delta_{a b} \delta_{\alpha \beta}, \\
& \left\{\Psi_{x}^{i a \alpha}, \Psi_{y}^{j b \beta}\right\}=\left\{\Psi_{x}^{i a \alpha \dagger}, \Psi_{y}^{j b \beta \dagger}\right\}=0,
\end{aligned}
$$

where $(i, j),(a, b)$ and $(\alpha, \beta)$ are color, flavor and Dirac indices, respectively. The generators of
$S U(N)$ gauge transformation take the form

$\vec{G}_{x}=\sum_{\mu}\left(\vec{R}_{x-\hat{\mu}, \mu}+\vec{L}_{x, \mu}\right)+\Psi_{x}^{\dagger} \vec{\lambda} \Psi_{x}$.

The dimensional reduction of fermions is a subtle issue. If one would use standard antiperiodic boundary conditions, familiar from thermodynamics, also in the extra dimension, the Matsubara modes, $p_{5}=2 \pi\left(n_{5}+\frac{1}{2}\right) / \beta$, would lead to a short $O(\beta)$ correlation length of the dimensionally reduced fermion. On the other hand, the gluon dynamics happens at distance scales which are exponentially large in $\beta$. As we have seen, $\beta$ acts as the lattice spacing of the dimensionally reduced effective Wilsonian theory. Fermions with antiperiodic boundary conditions in the fifth direction would hence remain at the cut-off and the dimensionally reduced theory would still be a Yang-Mills theory without quarks. Of course, there is no need to use antiperiodic boundary conditions for the fermions, because the additional dimension is not Euclidean time. When one uses periodic boundary conditions, a Matsubara mode, $p_{5}=0$, arises, and the quarks survive dimensional reduction. However, we then again face the fine-tuning problem of Wilson fermions.

The fine-tuning problem has been solved very elegantly in Shamir's variant 25] of Kaplan's fermion proposal [26]. Kaplan realized that 5$\mathrm{d}$ fermions coupled to a 4-d domain wall develop a zero-mode bound to the wall. From the point of view of the dimensionally reduced theory, the zero-mode represents a 4 -d chiral fermion. In QCD, Shamir has used a simpler variant of Kaplan's method, which is formulated in a 5 -d slab of finite size $\beta$ with open boundary conditions for the fermions at the two sides. This geometry fits naturally with the D-theory construction of quantum link models. The partition function with open boundary conditions for the quarks and with periodic boundary conditions for the gluons takes the form

$Z=\operatorname{Tr}\langle 0|\exp (-\beta H)| 0\rangle$.

The trace extends over the gluonic Hilbert space only. Following Shamir, we decompose the quark 
spinor into left and right-handed components

$\Psi_{x}=\left(\begin{array}{l}\Psi_{R x} \\ \Psi_{L x}^{\dagger}\end{array}\right)$.

Open boundary conditions for the fermions correspond to taking the expectation value of $\exp (-\beta H)$ in the Fock state $|0\rangle$, which is annihilated by the right-handed $\Psi_{R x}$ and by the lefthanded $\Psi_{L x}$ 27]. Consequently, no left-handed quarks are present at the $x_{5}=0$ boundary, and no right-handed quarks exist at the $x_{5}=\beta$ boundary of the 5-d slab.

In the presence of fermions, the low-energy effective theory of the gluons (with $A_{5}=0$ ) is modified to

$$
\begin{aligned}
& S[\bar{\psi}, \psi, A]= \\
& \int_{0}^{\beta} d x_{5} \int d^{4} x\left\{\frac{1}{2 e^{2}}\left[\operatorname{Tr} F_{\mu \nu} F_{\mu \nu}+\frac{1}{c^{2}} \operatorname{Tr} \partial_{5} A_{\mu} \partial_{5} A_{\mu}\right]\right. \\
& \left.+\bar{\psi}\left[\gamma_{\mu}\left(A_{\mu}+\partial_{\mu}\right)+M+\frac{1}{c^{\prime}} \gamma_{5} \partial_{5}\right] \psi\right\} .
\end{aligned}
$$

The "velocity of light" $c^{\prime}$ of the quarks in the fifth direction is expected to be different from the velocity $c$ of the gluons, because in D-theory there is no symmetry between the four physical spacetime directions and the extra fifth direction. This is no problem, because we are only interested in the 4-d physics after dimensional reduction.

Due to confinement, after dimensional reduction the gluonic correlation length is exponentially large, but not infinite. As explained in ref. 近, the same is true for the quarks, but for a different reason. Already free quarks pick up an exponentially small mass due to tunneling between the two boundaries of the 5-d slab. This mixes left- and right-handed states, and thus breaks chiral symmetry explicitly. The tunneling correlation length of the quarks is given by $2 M \exp (M \beta)$. This allows an elegant solution of the notorious fine-tuning problem of the fermion mass $M$. In D-theory the gluon dynamics of quantum link QCD in the chiral limit takes place at a length scale

$\xi \propto \exp \left(\frac{24 \pi^{2} \beta}{\left(11 N-2 N_{f}\right) e^{2}}\right)$,

which is determined by the 1-loop coefficient of the $\beta$-function of QCD with $N_{f}$ massless quarks and by the 5 -d gauge coupling $e$. Now one simply chooses

$M>\frac{24 \pi^{2}}{\left(11 N-2 N_{f}\right) e^{2}}$,

such that the chiral limit is reached automatically when one approaches the continuum limit by making $\beta$ large. This requires no fine-tuning.

\section{Conclusions}

We have seen that D-theory provides a rich structure, which allows us to formulate quantum field theories in terms of discrete variables quantum spins and quantum links. Dimensional reduction of discrete variables is a generic phenomenon. In $(d+1)$-dimensional quantum spin models with $d \geq 2$, for example, it occurs because of spontaneous symmetry breaking, while in $(4+1)$-dimensional non-Abelian quantum link models it is due to the presence of a 5-d massless Coulomb phase. The inclusion of fermions is very natural when one follows Shamir's variant of Kaplan's fermion proposal. In particular, the fine-tuning problem of Wilson fermions is solved very elegantly by going to five dimensions.

It is remarkable that D-theory treats bosons and fermions on an equal footing. Both are formulated in a finite Hilbert space per site, both require the presence of an extra dimension, and both naturally have exponentially large correlation lengths after dimensional reduction. The discrete nature of the fundamental variables makes D-theory attractive, both from an analytic and from a computational point of view. On the analytic side, the discrete variables allow us to rewrite the theory in terms of fermionic rishon constituents of the bosonic fields. This may turn out to be useful when one studies the large $N$ limit of various models. In particular, one can now carry over powerful techniques developed for condensed matter systems (like the quantum Heisenberg model) to particle physics. This includes the use of very efficient cluster algorithms, which has the potential of dramatically improving numerical simulations of lattice field theories.

In D-theory the classical fields of ordinary quantum field theory arise via dimensional re- 
duction of discrete variables. This requires specific dynamics - namely a massless theory in one more dimension. In general, the verification of this basic dynamical ingredient of D-theory requires non-perturbative insight - for example, via numerical simulations or via the large $N$ limit. Thus, the connection to ordinary field theory methods - in particular, to perturbation theory - is rather indirect. This could be viewed as a potential weakness, for example, because it seems hopeless to do perturbative QCD calculations in the framework of D-theory. However, the fact that perturbative calculations are virtually impossible may imply that non-perturbative calculations are now easier. After all, D-theory provides an additional non-perturbative microscopic structure underlying Wilson's lattice theory. Our hope is that this structure will help us to better understand the non-perturbative dynamics of quantum field theories.

\section{Acknowledgments}

Quantum link models were developed together with S. Chandrasekharan, and extended to quantum link QCD and D-theory in collaboration with R. Brower. The condensed matter aspects of this work have been investigated together with $\mathrm{B}$. B. Beard, R. J. Birgeneau, V. Chudnovsky and M. Greven. The other members of the MIT D-theory collaboration, who have significantly contributed to the research presented here, are D. Chen, J. Cox, K. Holland, B. Scarlett, B. Schlittgen and A. Tsapalis. I thank all these people for a most enjoyable collaboration. The work described here is supported in part by funds provided by the U.S. Department of Energy (D.O.E.) under cooperative research agreement DE-FC02-94ER40818, as well as by the A. P. Sloan foundation.

\section{REFERENCES}

1. D. Horn, Phys. Lett. 100B (1981) 149.

2. P. Orland and D. Rohrlich, Nucl. Phys. B338 (1990) 647.

3. S. Chandrasekharan and U.-J. Wiese, Nucl. Phys. B492 (1997) 455.
4. R. Brower, S. Chandrasekharan and U.-J. Wiese, hep-th/9704106.

5. B. B. Beard, R. Brower, S. Chandrasekharan, D. Chen, A. Tsapalis and U.-J. Wiese, Nucl. Phys. B (Proc. Suppl.) 63A-C (1998) 775.

6. B. B. Beard, S. Chandrasekharan, A. Tsapalis, three contributions to these proceedings.

7. T. Barnes, Int. J. Mod. Phys. C2 (1991) 659.

8. U.-J. Wiese and H.-P. Ying, Z. Phys. B93 (1994) 147.

9. B. B. Beard and U.-J. Wiese, Phys. Rev. Lett. 77 (1996) 5130.

10. P. Hasenfratz and H. Leutwyler, Nucl. Phys. B343 (1990) 241.

11. N. D. Mermin and H. Wagner, Phys. Rev. Lett. 17 (1966) 1133;

S. Coleman, Commun. Math. Phys. 31 (1973) 259.

12. P. Hasenfratz and F. Niedermayer, Phys. Lett. B268 (1991) 231.

13. P. Hasenfratz, M, Maggiore and F. Niedermayer, Phys. Lett. B245 (1990) 522;

P. Hasenfratz and F. Niedermayer, Phys. Lett. B245 (1990) 529.

14. S. Chakravarty, B. I. Halperin and D. R. Nelson, Phys. Rev. B39 (1989) 2344.

15. H. G. Evertz, G. Lana and M. Marcu, Phys. Rev. Lett. 70 (1993)

16. B. B. Beard, R. J. Birgeneau, M. Greven and U.-J. Wiese, Phys. Rev. Lett. 80 (1998) 1742.

17. U. Wolff, Phys. Rev. Lett. 62 (1989) 361; Nucl. Phys. B334 (1990) 581.

18. F. D. M. Haldane, Phys. Lett. 93A (1983) 464; Phys. Rev. Lett. 50 (1983) 1153.

19. H. Bethe, Z. Phys. 71 (1931) 205.

20. E. H. Lieb, T. Schultz and D. J. Mattis, Ann. Phys. 16 (1961) 407;

I. Affleck, T. Kennedy, E. H. Lieb and H. Tasaki, Phys. Rev. Lett. 59 (1987) 799; Commun. Math. Phys. 115 (1988) 477.

21. R. Botet, R. Jullien and M. Kolb, Phys. Rev. B30 (1984) 215;

J. B. Parkinson and J. C. Bonner, Phys. Rev. B32 (1985) 4703;

M. P. Nightingale and H. W. J. Blöte, Phys. Rev. B33 (1986) 659;

U. Schollwöck and T. Jolicouer, Europhys. 
Lett. 30 (1995) 493.

22. I. Affleck, Les Houches Summer School, Session XLIX, edited by E. Brezin and J. ZinnJustin (North Holland, Amsterdam, 1988), p. 563.

23. W. Bietenholz, A. Pochinsky and U.-J. Wiese, Phys. Rev. Lett. 75 (1995) 4524.

24. M. Creutz, Phys. Rev. Lett. 43 (1979) 553.

25. Y. Shamir, Nucl. Phys. B406 (1993) 90.

26. D. B. Kaplan, Phys. Lett. B288 (1992) 342.

27. V. Furman and Y. Shamir, Nucl. Phys. 439 (1995) 54. 